Castro, Constanza; "La dinámica interna de la Unión Cívica Radical durante la recuperación democrática. Organización partidaria, dirigentes y prácticas políticas (Córdoba, 1983-1987)". Cuadernos del Ciesal, Rosario, UNR, $\mathrm{N}^{\mathrm{o}}$ 20, 2021, pp. 1-27, ISSNe 1853-8827. https://cuadernosdelciesal.unr.edu.ar/index.php/inicio

\title{
La dinámica interna de la Unión Cívica Radical durante la recuperación democrática. Organización partidaria, dirigentes y prácticas políticas (Córdoba, 1983-1987)
}

\author{
Constanza Castro ${ }^{(*)}$
}

\begin{abstract}
Resumen
El trabajo examina la dinámica intrapartidaria de la UCR en la provincia de Córdoba a la luz del estudio de sus corrientes internas, las prácticas políticas en relación a la selección de sus candidatos y su actuación en las elecciones internas. El análisis se detiene particularmente en la reorganización partidaria previa a las elecciones de 1983 y los acuerdos entre líneas internas. Luego, examina las principales tensiones que atravesó el partido, organizadas en tres grandes ejes: la relación entre partido y gobierno, la inserción del radicalismo cordobés en la estructura nacional y; por último-trasversal a los dos primeros- los enfrentamientos entre las fracciones internas que se intensificaban en coyunturas electorales.
\end{abstract}

Palabras clave: Reconstrucción democrática; Partidos políticos; Unión Cívica Radical.

The internal dynamics of the Unión Cívica Radical during the democratic recovery. Party organization, leaders and political practices (Córdoba, 1983-1987)

\section{Abstract}

The work examines the intraparty dynamics of the UCR in the province of Córdoba in light of the study of its internal currents, the political practices in relation to the selection of its candidates and its performance in internal elections. The analysis focuses particularly on the party reorganization prior to the 1983 elections and the agreements between internal lines. Then, it examines the main tensions that the party went through, organized in three main axes: the relationship between party and government, the insertion of Cordovan radicalism in the national structure and; lastly - across the first two - the confrontations between the internal fractions that intensified at electoral junctures.

Key Words: Democratic reconstruction; Political parties; Unión Cívica Radical.

\footnotetext{
(*) Licenciada y profesora en Historia por la Universidad Nacional de Mar del Plata (UNMDP). Instituto de Investigaciones sobre Sociedades, Territorios y Culturas de la Universidad Nacional de Mar del Plata / Consejo Nacional de Investigaciones Científicas y Técnicas (CONICET), Argentina. Mail: constanzacastro2593@gmail.com ORCID: https://orcid.org/0000-0002-3901-7243
} 
"La dinámica interna de la Unión Cívica Radical durante la recuperación democrática. Organización partidaria, dirigentes y prácticas políticas (Córdoba, 1983-1987)"

\section{La dinámica interna de la Unión Cívica Radical durante la recuperación democrática. Organización partidaria, dirigentes y prácticas políticas (Córdoba, 1983-1987)}

\section{Introducción}

En 1983, la recuperación democrática fue de la mano del ascenso de la Unión Cívica Radical (UCR) que logró -en elecciones libres y sin proscripciones- alcanzar la primera magistratura. La victoria tuvo mayor impacto considerándose que el triunfo también se replicó en la provincia de Buenos Aires, principal bastión electoral del justicialismo. Menos sorprendente resultó en la provincia de Córdoba, distrito con una fuerte cultura política anclada en la tradición radical desde el gobierno de Amadeo Sabattini (Tcach, 1991). Allí, Eduardo Angeloz junto a Edgardo Grosso fueron electos gobernador y vicegobernador respectivamente.

Se ha afirmado que, desde esa elección, el sistema de partidos se configuró en base a una reprovincialización de la política argentina (Cavarozzi, 2006). Las provincias se constituyeron en escenarios privilegiados para forjar carreras políticas ascendentes que posicionaron a los gobernadores en la competencia por el ejecutivo nacional. La combinación en el control de recursos simbólicos, burocráticos y materiales operaron en beneficio de la consolidación de sus liderazgos (De Luca, Jones y Tula, 2008:84).

En diálogo con los análisis realizados -a nivel nacional- sobre la UCR durante los años ochenta (Persello, 2007; Delgado, 2010), que han destacado procesos generales que, sin desconocer las diferencias entre fracciones y en escenarios diversos, ofrecen una visión de conjunto y abren pistas para realizar indagaciones más específicas, el objetivo de este trabajo radica en examinar la dinámica partidaria de la UCR en la provincia de Córdoba a la luz del estudio de sus corrientes internas, las prácticas políticas en relación a la selección de sus candidatos y su actuación en las elecciones internas. Consideramos a la UCR como un sistema compuesto por subunidades, articuladas entre sí, entendidas como fracciones, en tanto están estructuradas desde un vértice y cuya organización contempla la elección de sus autoridades, la celebración de reuniones o congresos y el impulso de sus candidatos a cargos electivos (Sartori, 2005 [1976]:106-108).

Sostenemos que el radicalismo cordobés presentó una dinámica partidaria con importante dosis de informalidad que permitió a sus fracciones desarrollar su propio juego político valiéndose de los recursos a disposición. Las fracciones mayoritarias que disputaron el control de la organización fueron Línea Córdoba y el Movimiento de Renovación y Cambio (MRyC). Si bien esta última experimentó un crecimiento notable como correlato de la adhesión a la figura del entonces presidente, no alcanzó a contrarrestar el predominio de Línea Córdoba, cuyo éxito se explica en la construcción de un proyecto político partidario caracterizado por la recuperación de su tradición histórica y cierto pragmatismo en su accionar político. 


\section{Constanza Castro}

El trabajo consta de dos partes. En la primera se presentan las corrientes internas que protagonizaron la escena partidaria nacional y provincial, deteniéndose en la reorganización de la actividad partidaria durante la última apertura democrática y las negociaciones internas que atravesaron ese proceso. Por último, examina los principales problemas y las tensiones que caracterizaron al partido una vez que accedió al ejecutivo provincial.

\section{El radicalismo cordobés en la interna partidaria. La selección de candidatos para el ejecutivo nacional}

En vista de la normalización partidaria, Línea Córdoba y el MRyC, que eran las fracciones más gravitantes en el orden provincial, reeditaron la alianza que protagonizaron una década atrás. ${ }^{1}$ En esta ocasión, el entendimiento trascendió el orden provincial en incluyó a Víctor Martínez como vicepresidente.

La celeridad en la definición del binomio presidencial actuó en beneficio del alfonsinismo que instaló tempranamente en la escena intrapartidaria sus aspiraciones y obligó a Línea Nacional vertiente identificada con el histórico conductor del partido, Ricardo Balbín- a acelerar sus definiciones. La fórmula se definió en noviembre de 1982 cuando el líder del MRyC envió a la provincia mediterránea una comitiva para negociar la adhesión de Línea Córdoba, acordándose la candidatura de Víctor Martínez a la vicepresidencia y la de Angeloz en la provincia (Frávega, 2006:331). A pesar de que el acuerdo ya había sido suscripto entre ambas fracciones y se había convocado a un acto de presentación, Línea Córdoba sometió -como mera formalidad- la alianza a consideración de su asamblea provincial. ${ }^{2}$ Tres días más tarde, en un multitudinario acto realizado en el estadio Luna Park que tuvo como únicos oradores a Alfonsín y Martínez, se oficializó la fórmula.

En contraste, la dispersión caracterizó a Línea Nacional, cuyo nucleamiento tenía dos orientaciones representadas por los dirigentes Fernando De la Rúa y Juan Carlos Pugliese que realizaron por separado sus deliberaciones. Los seguidores del primero proclamaron su precandidatura presidencial; mientras que los segundos intentaron evitar la confrontación y, como muestra de ello, Pugliese declinó su precandidatura. ${ }^{3}$ Tanto Pugliese como otros dirigentes balbinistas pretendían impulsar una fórmula que incluyera a De la Rúa secundando a Alfonsín. Sin embargo, ambos dirigentes se mostraron renuentes a esa posibilidad.

\footnotetext{
${ }^{1}$ Cuando Raúl Alfonsín compitió por el control de la UCR escindiéndose del balbinismo, estableció acuerdos con distintos dirigentes y agrupamientos provinciales. En Córdoba, el Movimiento Nuevo a cuyo frente se encontraba Angeloz, acompañó al binomio Alfonsín- Conrado Storani y aportó un cuantioso caudal de votos. Al poco tiempo, los alfonsinistas que participaron del Movimiento Nuevo conformaron el MRyC en la provincia, cuya titularidad recayó en Carlos Becerra. Esa fuga reconfiguró el Movimiento Nuevo que a partir de entonces pasó a denominarse Línea Córdoba.

2 "Aprueban radicales de Córdoba una alianza electoral interna", El Día, La Plata, 5/12/1982, p.4.

3 “Enfrentamiento en Línea Nacional”, El Día, La Plata, 21/12/1982, p. 1 y 4.
} 
“La dinámica interna de la Unión Cívica Radical durante la recuperación democrática. Organización partidaria, dirigentes y prácticas políticas (Córdoba, 1983-1987)"

Frente a una posible revisión, Martínez afirmó que las "vacilaciones" de Línea Nacional no pertenecían a su movimiento y que, de producirse algún tipo de alteración "no se puede retirar una persona, sino que se tendría que retirar la Línea Córdoba". En la misma sintonía se expresó Angeloz que argumentaba la inviabilidad de la propuesta dado que "el radicalismo más prestigioso del país" se hallaba en Córdoba, a la vez que afirmaba que la fórmula tanto a nivel nacional como provincial estaba "conversada y convenida" y que luego de las instancias internas sería convalidada. ${ }^{4}$

Lejos de alcanzar un entendimiento, el 4 de febrero de 1983 el binomio De la Rúa- Carlos Perette fue consagrado oficialmente en un acto realizado a fines del mes de marzo. ${ }^{5}$ No obstante, a medida que comenzaron a celebrarse las elecciones internas entre los meses de junio y julio, y aun cuando en algunas provincias no se habían sustanciado, era evidente el avance del alfonsinismo que ya contaba con la mayoría de delegados para imponerse en el Comité Nacional. En consecuencia, De la Rúa retiró su precandidatura y aceptó competir como senador por la Capital Federal. ${ }^{6}$

\section{El acuerdo en la provincia y las fuerzas en disputa}

La inclusión de un representante de Línea Córdoba en la fórmula presidencial hacía necesario extender los acuerdos en el orden provincial, más allá de la definición de la indiscutida candidatura a gobernador de Angeloz como resultado de su trayectoria y liderazgo interno. ${ }^{7} \mathrm{~A}$ diferencia del crecimiento exponencial que experimentó el alfonsinismo en varios distritos, en Córdoba la hegemonía fue capitalizada por Línea Córdoba que reunía dirigentes con amplia trayectoria y controlaba la maquinaria electoral de la provincia. Por el contrario, el MRyC organizado poco antes de la interrupción constitucional de 1976 y que había sufrido la pérdida de Carlos Becerra, uno de sus referentes más gravitantes, no contaba con una estructura sólida ni con dirigentes de nutridas trayectorias que disputaran la representación a la poderosa Línea Córdoba. Quienes se destacaron como dirigentes políticos del alfonsinismo cordobés -en su mayoría- habían abandonado recientemente la militancia universitaria y su experiencia se remitía a la ocupación de cargos en los órganos del cogobierno universitario o partidarios en el ámbito de la Juventud Radical. En consecuencia, se afianzaron en la escena política partidaria una vez que el radicalismo accedió al ejecutivo provincial desde los espacios que ocuparon como socios minoritarios de la alianza o al amparo de las gestiones alentadas por Alfonsín.

\footnotetext{
4 "El Dr. Pugliese extiende su gestión unificadora a Córdoba", La Nación, 7/1/1983, p. 5.

5 "El balbinismo consagró la fórmula De la Rúa- Perette", La Nación, 5/2/1983, p. 1 y 12.

6 "Presentaron la renuncia a sus candidaturas De la Rúa y Perette “, Clarín, 19/7/1983, p. 3.

7 Angeloz se desempeñó como presidente del Comité Capital de la Juventud (1953), presidente del Comité Capital del partido (1963) y del Comité Central de la Provincia entre 1972 y 1974, con mandato prorrogado hasta 1983. En la arena legislativa fue Senador Provincial (1963-1966) y Senador Nacional (1973-1976).
} 


\section{Constanza Castro}

El balbinismo -que también era minoritario en la provincia- sufrió los efectos de las tensiones protagonizadas en el orden nacional que incidieron aún más en su desarticulación. En concomitancia a la disputa protagonizada por los dirigentes De la Rúa y Pugliese, dos grupos que respondían a sus liderazgos contendieron por la representación balbinista. Por un lado, el sector que reconocía a Pugliese se denominó Línea Nacional Federalista y se encolumnó tras el dirigente Horacio García, manifestándose a favor de la postulación de Alfonsín como presidente, pero manteniendo su autonomía para la elección de autoridades partidarias. Por otra parte, quienes se referenciaron con De la Rúa, se dividieron en dos núcleos: uno liderado por Mario Roberto, y otro por Luis Urtebey. En un plenario realizado en la ciudad de Villa María, se eligió como titular de la vertiente a Mario Roberto, ausentándose el sector de Urtebey que pasó a denominarse Línea Nacional Córdoba Autónoma. ${ }^{8}$

\section{El enfrentamiento por la conducción partidaria}

La competencia por la renovación de las autoridades partidarias fue establecida para el día 10 de julio de 1983. El enfrentamiento se dirimió entre dos listas: la $\mathrm{N}^{\circ} 1$, representada por miembros de Línea Córdoba y el MRyC que impulsaba a Roberto Llorens como candidato a presidir el comité provincial; y la $\mathrm{N}^{\circ} 2$, que representaba una alianza coyuntural entre el Movimiento Sabattinista de Córdoba -identificado con el Movimiento de Afirmación Yrigoyenista (MAY)y Línea Nacional, cuya candidatura descansó en Alfredo Morello.

La capital provincial fue el escenario más disputado y el que mayor grado de fragmentación evidenció dado que las alianzas no se replicaron en todas en las secciones por igual y se sumaron a la contienda otros nucleamientos. La lista $\mathrm{N}^{\circ} 1$ impulsó la postulación de Alfredo Orgaz como candidato a presidir el comité capitalino, mientras que la $\mathrm{N}^{\circ} 2$ hizo lo propio con la nominación de Aldo Villarreal. Pese a esas divisiones, la lista encabezada por Llorens se impuso ampliamente en todas las secciones de la capital, incluso en las que no prosperó el acuerdo. En el interior provincial las listas fueron más uniformes, con la salvedad de Villa Carlos Paz donde el alfonsinismo también decidió concurrir separado de Línea Córdoba. Si bien esta última ganó la contienda, la diferencia fue más ajustada en comparación con la altísima ventaja que obtuvo en otros espacios en los que triunfó ampliamente, evidenciándose su predominio territorial a nivel provincial. ${ }^{9}$

La Lista $\mathrm{N}^{\circ} 1$ se impuso no solo en todas las secciones de la capital sino también en la mayoría de departamentos y circuitos del interior provincial. Llorens obtuvo poco más del $90 \%$ y Orgaz alcanzó casi el 85\% consagrándose presidente del comité provincial y capital respectivamente.

\footnotetext{
8 “Nuevas autoridades de Línea Nacional Córdoba", La Nación, 16/5/1983, p. 6.

9 "Se cumplen las internas del radicalismo provincial", La Voz del Interior (LVI), 10/7/1983, p.9.
} 
“La dinámica interna de la Unión Cívica Radical durante la recuperación democrática. Organización partidaria, dirigentes y prácticas políticas (Córdoba, 1983-1987)"

Estos resultados otorgaron mayoría absoluta a la alianza entre Línea Córdoba y el MRyC en la composición del Comité Provincial y entre los delegados al Comité Nacional. ${ }^{10}$

\section{La definición de las candidaturas y el triunfo electoral}

Pese al intento de impulsar una fórmula que disputara la representación radical para el ejecutivo provincial y en consonancia con la declinación del binomio presidencial De la Rúa- Perette, el sector balbinista y sus aliados no presentaron candidatos para competir internamente de cara a las elecciones generales del 30 de octubre de 1983. La contundente derrota para la renovación de autoridades partidarias evidenció el paupérrimo desempeño del balbinismo en los escasos espacios en los que pudo conformar listas y lanzarse a la competencia.

La selección de las candidaturas finales constituyó una mera instancia formal dado que se habían definido con antelación. La postulación de Angeloz fue completada con la designación de Edgardo Grosso como compañero de fórmula. ${ }^{11}$ Una vez consensuada su postulación, a través de una asamblea realizada en la Capital, se impulsó su proclamación que fue ratificada al día siguiente- en la reunión provincial del nucleamiento interno de Línea Córdoba. ${ }^{12}$

Luego de la convocatoria del Congreso Partidario Provincial, la Junta Electoral proclamó las nominaciones de las listas que no habían tenido contrincantes, entre ellos a Angeloz y Grosso como candidatos a gobernador y vicegobernador respectivamente; y a Ramón B. Mestre como candidato a intendente por la Capital provincial. Ambas fórmulas triunfaron en las elecciones generales del 30 de octubre de 1983 imponiéndose ampliamente contra sus adversarios justicialistas. La contundente victoria se reflejó en la composición de ambas cámaras legislativas, con amplia mayoría radical: en el senado, la UCR se alzó con 31 bancas y el PJ con apenas 6; en diputados, 24 lugares correspondieron al radicalismo y 12 al peronismo. En la arena nacional, Córdoba aportó once diputados por la UCR y seis por el PJ; mientras que, para electores de presidente, 23 correspondieron a la UCR y 17 al PJ.

\section{El radicalismo cordobés en la encrucijada: desafíos y tensiones partidarias}

Luego del contundente triunfo, el radicalismo asumió su nuevo rol como partido de gobierno. En el orden partidario atravesó una serie de tensiones que se manifestaron con variada intensidad en momentos específicos. Ciertamente, la dinámica del radicalismo cordobés durante

\footnotetext{
${ }^{10}$ Listas de autoridades completas en “Asumen el miércoles autoridades de la UCR", LVI, 23/7/1983, p.9.

${ }^{11}$ Había sido intendente de General Cabrera (1963-1967) y senador provincial (1973-1976). A su nominación contribuyó la renuncia de Jorge Cendoya, dirigente de la ciudad de Río Cuarto que durante la dictadura militar estuvo detenido, imputado por ilícitos en su desempeño como presidente del Banco Popular de Río Cuarto. Ante ese antecedente que podría ser contraproducente para la imagen del partido, se propuso a Grosso (Frávega, 2006:331).

12 "Candidato a vicegobernador", La Nación, 18/3/1983, p. 8.
} 


\section{Constanza Castro}

el primer gobierno democrático estuvo atravesado por tres grandes ejes: la relación entre partido y gobierno, la inserción del radicalismo cordobés en la estructura nacional y por último trasversal a los dos primeros- los enfrentamientos entre las fracciones internas que se intensificaban en coyunturas electorales.

\section{a- ¿Un radicalismo movilizado? La relación partido- gobierno}

La relación entre gobierno y partido fue una de las preocupaciones que se manifestó con fuerza en la primera etapa de gobierno. Las críticas iníciales fueron formuladas por los alfonsinistas que, ante el predominio de dirigentes de Línea Córdoba en la mayor parte de los órganos partidarios, objetaron el lánguido funcionamiento y la escasa movilización partidaria. Los cuestionamientos afloraron tempranamente cuando las autoridades partidarias prohibieron la apertura del Centro Cívico "Carlos Becerra” impulsado por un grupo de jóvenes del MRyC. E1 vicepresidente del comité sancionó a los dos militantes que se desempeñaban como secretarios al argumentar que la carta orgánica impedía la actividad de aquellos centros que no contaban con la aprobación de las autoridades. ${ }^{13}$

Los dirigentes sancionados y los diputados provinciales del MRyC objetaron las atribuciones del comité para sancionar a los militantes y manifestaron que el gobierno necesitaba "un partido activo y movilizado" con lo cual condenaban las actitudes que - a su entender- obstaculizaban el crecimiento del radicalismo y coartaban la confianza de la ciudadanía. ${ }^{14}$ En la misma línea, el balance partidario realizado por el MRyC durante el primer año de gobierno resultó infructuoso. El presidente del Comité Provincial de la Juventud Radical, Mario Negri, fue quien expuso la lectura realizada por el alfonsinismo, a la vez que formuló sus propios cuestionamientos:

“(...) el radicalismo de Córdoba, fundamentalmente su conducción, no ha reflejado la madurez suficiente para acompañar la buena gestión de gobierno que se ha hecho en la provincia (...) Creemos que se ha entretenido en las pequeñas cosas de la interna y en algunos casos ha permitido la desmovilización del partido a pesar de los inmensos esfuerzos de vastos sectores por encima de las líneas internas que han apostado permanentemente a un partido de puertas abiertas y no de puertas cerradas". ${ }^{15}$

\footnotetext{
13 "Divergencias partidarias", LVI, 17/5/1984, p.9.

14 "UCR: enfoques opuestos sobre centros cívicos", $L V I, 18 / 5 / 1984$, p.7.

15 “Criticó Negri la conducción del radicalismo provincial”, LVI, 11/2/1985, p. 7.
} 
“La dinámica interna de la Unión Cívica Radical durante la recuperación democrática. Organización partidaria, dirigentes y prácticas políticas (Córdoba, 1983-1987)"

Las declaraciones de Negri provocaron el repudio de vastos dirigentes del partido que delegaron la réplica en Héctor Sander, titular del bloque de senadores, quien argumentó que el partido se había movilizado a través de los organismo correspondientes; a la vez, objetaba la doble función de Negri en el ámbito de la Juventud Radical que, además de ser presidente del Comité Provincial se desempeñaba como vicepresidente del mismo organismo a nivel nacional, aspecto que -en su visión- imposibilitaba un análisis preciso y objetivo. ${ }^{16}$ Meses más tarde, con motivo de la elección interna protagonizada en la Capital provincial, el mismo Sander evaluó como "un fracaso" la gestión del Comité partidario en el distrito. En la misma línea se manifestó Fernando Montoya -presidente del concejo deliberante capitalino- que marcaba la confusión existente entre partido y gobierno, y señalaba que correspondía al partido apoyar la gestión de gobierno, pero también ser el control del cumplimiento de los postulados de la plataforma y los principios partidarios. ${ }^{17}$

Hacia 1985 cuando el MRyC comenzó a conquistar mayores espacios partidarios, electivos y de gestión, las críticas fundadas en la desmovilización y la confusión entre partido y gobierno fueron soslayadas; mientras que esos argumentos fueron apropiados por aquellos sectores que se lanzaban a la competencia interna.

\section{b- La inserción del radicalismo en la estructura nacional}

La preocupación por ocupar espacios en la estructura partidaria nacional tomó fuerza luego de la renovación de autoridades del Comité Nacional realizada a comienzos de diciembre de 1985. La caducidad del mandato de Víctor Martínez como vicepresidente dejaba al distrito sin representación, motivo por el cual Línea Córdoba aspiró a conquistar la vicepresidencia tercera impulsando a Edgardo Grosso, quien reclamaba su inclusión por la gravitación electoral de la provincia. ${ }^{18}$

Sin embargo, las aspiraciones se vieron frustradas frente a los santafesinos que impusieron en la vicepresidencia tercera a Aníbal Reinaldo. Con ello, el distrito cordobés quedó relegado a la ocupación de una secretaria a cuyo mando estuvo Grosso. ${ }^{19}$ Como consecuencia de estos movimientos que evidenciaban una relativa marginalidad en los estratos superiores del partido, Línea Córdoba impulsó un replanteo sobre su inserción en la estructura nacional, cuyo argumento se apoyó en la fusión del nucleamiento dentro del MRyC. Sin duda, a esta iniciativa también contribuyó el viraje del gobierno nacional en los posicionamientos esgrimidos durante el discurso pronunciado por Alfonsín en Parque Norte el 1 de diciembre de 1985, especialmente

\footnotetext{
16 "Las críticas de Negri no reflejan la realidad", $L V I, 18 / 2 / 1985$, p. 7.

17 "La interna radical", $L V I, 17 / 7 / 1985$, p. 7.

18 "Fundamentó Grosso aspiración del radicalismo cordobés", LVI, 29/11/1985, p. 9.

19 “Composición de la mesa directiva”, La Nación, 2/12/1985, p. 12.
} 


\section{Constanza Castro}

en materia económica, ámbito en el que la noción de "modernización" cobró relevancia y acercaba a los dirigentes cordobeses.

Las deliberaciones en torno a la iniciativa unificadora se realizaron por separado hacia fines de ese mismo año. Reunido en la localidad de Isla Verde, el MRyC difundió un documento que manifestaba su renuencia a alcanzar la fusión y lo evidenciaba en su título: "Fuimos, somos y seguiremos siendo el Movimiento de Renovación y Cambio, expresión genuina de la Unión Cívica Radical”. En su argumento, diferenciaban entre las alianzas electorales y el contenido programático, argumentando que su vinculación a Línea Córdoba respondía a la primera y por ello era imposible fusionarse. Por su parte, la dirigencia de Línea Córdoba se mantuvo incólume en afirmar "la necesidad de reorganizar el radicalismo cordobés para superar la estratificación partidaria". Consideraban la urgencia en impulsar "cambios partidarios" y una redefinición del perfil dirigida a alcanzar una consolidación con proyección nacional. ${ }^{20}$

La discusión cobró protagonismo en el bienio 1986-1987. En un primer momento, que se extiende hasta la primera mitad del año 1986, el debate giró en torno a dos ejes que imposibilitaron arribar a un consenso: por un lado, confrontaron dos lecturas sobre el pasado reciente del radicalismo en la provincia, particularmente sobre la alianza protagonizada para las elecciones internas de 1972; por otro lado, emergió como problema la cuestión del liderazgo: ante una eventual simbiosis entre ambos espacios, ¿quién ostentaría su representación? Con la figura de Carlos Becerra (h) en ascenso dada su gravitación nacional y el predominio ejercido por Angeloz a nivel provincial, ambos dirigentes eran presentados como antagónicos; por lo tanto, dirimir una futura conducción representaba un obstáculo insuperable.

En cuanto al primer eje, luego de un plenario realizado en Cosquín, el MRyC difundió un nuevo documento titulado "Consolidación de la democracia en la Argentina", en el que reconocía a los dirigentes que se habían destacado en el distrito y se refería a las elecciones internas de 1972 afirmando que:

"un gran sector del radicalismo provincial adhiere circunstancialmente al MNRC en 1972, como oposición a la conducción que a nivel nacional desarrollaba Ricardo Balbín, sin compartir los contenidos de fondo ni las propuestas metodológicas (...) [ello desemboca en 1975] en la conformación de una línea provincial (Línea Córdoba) y solamente sirve a los fines de sus impulsores, incurriendo en el mismo error del viejo esquema partidario, tomando la vía personal de proyección de

20 “Delibera Renovación y Cambio y Línea Córdoba”, Clarín, 22/12/1985, p. 12. 
"La dinámica interna de la Unión Cívica Radical durante la recuperación democrática. Organización partidaria, dirigentes y prácticas políticas (Córdoba, 1983-1987)"

manera tal que, debajo de ellos, no existen fuentes generadoras de políticas para el conjunto partidario". ${ }^{21}$

Contrariamente a esa lectura, los sectores juveniles de Línea Córdoba imprimieron mayor celeridad a su incorporación al MRyC, interpretándose como un anticipo de lo que iba a ocurrir en la plana mayor. El traspaso fue formalizado durante el Segundo Seminario Provincial de la Juventud Radical de Línea Córdoba realizado en Villa Allende el 19 de julio de 1986 y decidido por aclamación. El documento aprobado durante la reunión señalaba:

"el radicalismo de Córdoba supo interpretar el proyecto dinamizador de los principios de Alem e Yrigoyen planteado en 1972 (...) se trata de una reinserción basada en el testimonio político que lleva adelante Eduardo Angeloz, al interpretar fielmente el proyecto político impulsado por el presidente Alfonsín desde Parque Norte, en diciembre de 1985, cuando fundamentó su convocatoria a la convergencia en los principios de la democracia participativa, ética de la solidaridad y modernización"22

A la vez que evidencia la influencia del discurso de Parque, la alusión a "la reinserción" remitía a la confluencia protagonizada para las elecciones internas de 1972. En la misma línea, Orgaz expresó que correspondía al MRyC la realización de un replanteo en cuanto a la fusión dado que significaba "volver a lo que siempre fuimos". ${ }^{23}$ En cuanto a los liderazgos y la conducción provincial, existió un fuerte cuestionamiento de algunos militantes de Línea Córdoba hacia Carlos Becerra, quien se manifestaba en contra del "oportunismo electoral" y calificaba de "gatopardeada" las ansias de traspaso. Becerra cuestionaba: “¿cómo las autoridades de Línea Córdoba le van a explicar a sus militantes que ellos son los auténticos dirigentes de Renovación y Cambio y que quienes actualmente conducen esa corriente son unos tramposos porque nunca lo fueron?" 24

Los primeros embates de Línea Córdoba procedieron del sector juvenil cuando afirmaron que Becerra no era el representante de Alfonsín en la provincia y, por lo tanto, desconocían su liderazgo. ${ }^{25} \mathrm{La}$ réplica fue difundida por la Junta Provincial del MRyC en un documento denominado "Hay que seguir los principios, no a los hombres". Allí, aceptaban la identificación del sector juvenil con su espacio, pero objetaban el cuestionamiento realizado sobre Becerra:

\footnotetext{
21 “Crítico documento de Renovación y Cambio", LVI, 24/2/1986, p. 1. El destacado es mío.

22 "La Juventud de Línea Córdoba se incorpora a Renovación y Cambio", LVI, 20/7/1986, p. 6. El destacado es mío.

23 “'Alianza Civil' en Córdoba”, Clarín, 12/10/1986, p. 8.

24 "Becerra", LVI, 8/5/1986, p. 9.

25 "'Becerra no es el hombre de Alfonsín en Córdoba”, LVI, 18/7/1985, p. 4.
} 


\section{Constanza Castro}

“evidentemente sería un hecho auspicioso que estos jóvenes abandonen un proyecto personalista como el de Línea Córdoba, agotado por falta de contenido político y cuyo nombre ahuyenta a los jóvenes, pues lo asocian con la falta de debate y el verticalismo interno (...) lo que nos extraña y lamentamos profundamente es que estos jóvenes de Línea Córdoba que aún no ingresaron a nuestro movimiento ya pretendan interpretarlo y dividirlo, y aún más cuestionar a su presidente, el secretario general de la Presidencia, Carlos Becerra que, vale la pena destacarlo, conduce al Movimiento Nacional de Renovación y Cambio". ${ }^{26}$

En vista de estas tensiones y las suscitadas con motivo de las negociaciones que enmarcaron la Convención Constituyente que tenía por fin reformar la constitución -encontrándose entre sus ejes principales la reelección-, la pretendida fusión quedó en suspenso hasta principios de 1987. De hecho, Línea Córdoba que había anunciado la convocatoria a un "cabildo abierto" en el que debatiría y sometería a votación su incorporación al MRyC, prorrogó en numerosas ocasiones su realización. Éste pudo llevarse a cabo una vez que las negociaciones internas entre las dos fracciones habían finalizado y el consenso en el reparto de los cargos partidarios y electivos conformaron las aspiraciones de ambas. En efecto, en la misma asamblea en la que se proclamó la candidatura de Angeloz a la reelección, previamente se decidió por votación la integración de Línea Córdoba al MRyC. Tanto Becerra como Angeloz convinieron en "aggiornar" el partido y colocarlo "a la altura de las circunstancias". ${ }^{27}$

\section{c- “Que se rompa pero no se doble”. Las elecciones internas y la selección de candidatos entre dos elecciones: 1985-1987}

La celebración de elecciones para la renovación de cargos legislativos y ejecutivos coincidieron con los comicios internos del partido que debían efectuarse cada dos años. En este período cobró centralidad Carlos Becerra a partir de su designación en febrero de 1986 como Secretario General de la Presidencia. ${ }^{28} \mathrm{Si}$ bien su nombramiento no generó un desequilibrio interno en beneficio de su movimiento, otorgó un mayor protagonismo a esa corriente y al dirigente que también se desempeñaba como titular de la Junta Provincial.

En relación a la dinámica interna del partido y la renovación de sus autoridades, en las dos elecciones podemos identificar un conjunto de tensiones en torno a la normativa que regía su

\footnotetext{
26 “Objetan cuestionamiento a Becerra", LVI, 20/7/1985, p. 6.

${ }^{27}$ La incorporación fue aprobada con 80 votos a favor y 4 en contra. "Fue formalizado el ensamble en el radicalismo provincial", LVI, 13/4/1987, p. 1 .

${ }^{28}$ Desde 1983 se desempeñó como Diputado Nacional por Córdoba.
} 
"La dinámica interna de la Unión Cívica Radical durante la recuperación democrática. Organización partidaria, dirigentes y prácticas políticas (Córdoba, 1983-1987)"

funcionamiento. Estas fueron sorteadas con prácticas informales que privilegiaron los acuerdos de cúpula. Los escollos partidarios a los que referimos son: el excesivo enfrentamiento que condujo a la creación de nuevos cargos partidarios para conformar a grupos internos, la incompatibilidad en el ejercicio de cargos partidarios y públicos; y la cláusula para la reelección de autoridades y candidatos que exigía alcanzar los dos tercios de los votos para acceder a una nueva nominación; reglamentándose los dos últimos casos en la Carta Orgánica del partido.

\section{La renovación partidaria y legislativa de 1985}

Todas las corrientes internas entendían la necesidad de alcanzar consenso para reforzar la imagen de unidad que el partido pretendía. En consecuencia, las vertientes mayoritarias realizaron asambleas para seleccionar los candidatos según la distribución que previamente habían acordado. Línea Córdoba demoró sus nominaciones, no sesionó hasta que no aseguró la asistencia de Angeloz, acción que evidenciaba su preeminencia en las resoluciones finales. Por su parte, el MRyC realizó una asamblea con el fin de seleccionar las nominaciones. Si bien para los cargos de mayor jerarquía partidaria estaban definidos los candidatos, no existió consenso en la designación de una sexta secretaría. El episodio tornó conflictiva la reunión y dio cuenta de la intensidad de los enfrentamientos para los cargos de menor jerarquía, poniendo en discusión los mecanismos de selección de las nominaciones. ${ }^{29}$

Superada la instancia de las asambleas, se reunió el Congreso Provincial de la UCR, cuya labor fundamental radicó en las prerrogativas partidarias que sancionó. Se aprobó la suspensión de las incompatibilidades en el desempeño de las funciones públicas y partidarias con vigencia por trescientos días, período en el que una comisión especial -designada por el mismo Congresodebía expedirse sobre ese asunto. ${ }^{30}$ La medida fue aprobada por un escaso margen de votos dado que contó con el aval de sesenta delegados, dos más de los necesarios, mientras que veintisiete se pronunciaron por el mantenimiento de la normativa enfatizando en el "carácter ético" de las incompatibilidades.

La oposición emergió con motivo de la disputa interna que comenzó a manifestarse en la Capital entre dos sectores de Línea Córdoba, evidenciándose una incipiente bifurcación: una, se referenciaba con el intendente Mestre y otra lo hacía con Angeloz. El primer sector fue liderado por Héctor Sander y reunió a dirigentes que en su mayoría se desempeñaban como funcionarios de gobierno y en el concejo deliberante de la Capital. El segundo sector fue orientado por Orgaz, quien señaló que no existían diferencias ideológicas sino políticas. Resaltaba las discrepancias argumentando que su espacio apoyaba "la acción del gobierno en los tres

\footnotetext{
29 “Candidatos", LVI, 21/6/1985, p. 1.

30 “Carrizo presidirá la UCR”, LVI, 23/6/1985, p. 9.
} 


\section{Constanza Castro}

órdenes", reconocía a Angeloz como "el conductor natural del radicalismo cordobés" y a Mestre como "un intendente más de todos los que tiene la UCR en el país". ${ }^{31}$

Ambos sectores se enfrentaron por la titularidad del Comité Capital y la nominación de las dos senadurías provinciales del distrito. El núcleo encabezado por Sander reunió la adhesión del MRyC “Auténtico” -escisión liderada por la diputada nacional Dolores Díaz de Agüero-, de los minoritarios MAY y Línea Nacional, junto al sector juvenil encabezado por Carlos Vicente que reunía a la mayoría de los militantes universitarios de Franja Morada. Propuso para ocupar la presidencia del Comité a Fernando Montoya y postuló la reelección de Sander y Contreras como senadores. Entre algunas de sus propuestas resaltaban la necesidad de una mayor apertura de los comités, criticaban "las nominaciones a dedo" sin intervención de los afiliados y enfatizaba en el carácter elitista de la actual conducción a la cual adjudicaban la pretensión de "apartar y sectorizar". ${ }^{32}$ Por su parte, el sector liderado por Orgaz, que era reconocido por Angeloz y el MRyC, impulsó la reelección de ese dirigente como titular del Comité que debía alcanzar como mínimo los dos tercios de los votos para convalidar un nuevo periodo. Para las dos senadurías levantaban las candidaturas de Nilo Neder y Regino Maders.

La elección interna de la Capital era importantísima por la gravitación del distrito a nivel provincial ya que reunía a más de un tercio de los afiliados. ${ }^{33} \mathrm{La}$ breve disputa estuvo atravesada por acusaciones de adulteraciones en los padrones y "limpieza selectiva" por parte de la municipalidad respecto de la actividad proselitista realizada en la vía pública. Producida la contienda electoral, el sector de Orgaz se impuso sobre el de Sander. La proclamación como senadores de Neder y Maders se hizo efectiva pero, en el ámbito partidario, Orgaz obtuvo el $61 \%$ de los votos y -aun así- no logró reunir los dos tercios necesarios para acceder a la reelección. ${ }^{34}$ Bajo esa circunstancia, según la carta orgánica, el nuevo presidente del comité debía surgir de la primera reunión realizada entre los miembros electos. Sin embargo, los intentos estuvieron dirigidos a legitimar en el cargo a Orgaz y asegurar su continuidad. En reiteradas oportunidades se pretendió negociar un "acuerdo político"-tal como era definido por los mismos actores- que finalmente no arribó a buen puerto. ${ }^{35}$ Ante la urgencia de resolver la conducción partidaria por la inminente elección general de noviembre, la UCR se ajustó temporalmente a la normativa dispuesta por la carta orgánica y asumió la titularidad Mario Gutiérrez, miembro de Línea Córdoba y postulado inicialmente para la primera secretaría.

Pese a que el radicalismo obtuvo un contundente triunfo a nivel provincial y, en el plano partidario, contuvo las dificultades de manera efectiva ajustándose a la normativa, el conflicto

\footnotetext{
31 “Orgaz: reconocemos a Angeloz como conductor", LVI, 5/7/1985, p. 7.

32 "Por un radicalismo sin exclusiones", $L V I, 19 / 7 / 1985$, p. 5.

${ }^{33}$ En la Capital había 78.654 afiliados habilitados para la votación de un padrón provincial de 216.960. "Finalizó la campaña radical", $L V I, 20 / 7 / 1985$, p. 7.

34 "Amplio triunfo de Orgaz en la Capital", $L V I, 22 / 7 / 1985$, p. 1.

35 "Insistirán para que Orgaz presida el Comité Capital", LVI, 23/7/1985, p. 7.
} 
"La dinámica interna de la Unión Cívica Radical durante la recuperación democrática. Organización partidaria, dirigentes y prácticas políticas (Córdoba, 1983-1987)"

emergió nuevamente cuando Gutiérrez anunció con "carácter indeclinable" su renuncia. En una coyuntura poco propicia al entendimiento, dado que se avecinaba la Convención Constituyente y los vínculos entre Línea Córdoba y el MRyC se habían deteriorado, el accionar frente a la renuncia mostró las divergencias de las distintas fracciones. Por un lado, los miembros del Comité rechazaron la renuncia y se reunieron con Gutiérrez con el objetivo de declinar su posición; mientras que el MRyC avaló públicamente su decisión ya que, de hacerse efectiva, Miguel Ortiz Pellegrini, que se desempeñaba como vicepresidente primero del Comité capitalino, pasaba automáticamente a asumir la titularidad. Idéntico reconocimiento asumió la renuncia en los adversarios internos de Línea Córdoba y en algunos sectores juveniles. ${ }^{36}$

\section{La reforma constitucional y las elecciones de 1987}

Las elecciones realizadas en 1987 son indisociables de los movimientos partidarios ocurridos el año previo con motivo de la convocatoria para reformar la constitución. Impulsada por la Cámara de Senadores hacia fines de mayo de 1986, la posibilidad de habilitar la reelección del cargo ejecutivo se ubicó en el centro de las discusiones, en tanto Línea Córdoba aspiraba a retener la gobernación con Angeloz y el MRyC trataba de imponer la candidatura de Becerra.

Para alcanzar su objetivo, Línea Córdoba necesitaba un rápido tratamiento y su aprobación en la Cámara de Diputados, ámbito en el que la UCR contaba con mayoría propia. Sin embargo, en la distribución de legisladores dieciocho diputados pertenecían a Línea Córdoba y seis al MRyC, ecuación que dejaba sin quorum propio a los primeros. En consecuencia, la estrategia de los alfonsinistas consistió en retardar su discusión en el recinto a través del pedido de licencia de sus diputados. Sin negarse a la reforma, afirmaban que era necesario impulsar un profundo debate que incluyera a todos los sectores de la sociedad para no desembocar en un "mero oportunismo electoral". Demorándose su tratamiento, especulaban que vencidos los plazos para la presentación de las candidaturas y al amparo de la constitución vigente, imposibilitado de presentarse Angeloz a renovar mandato, la postulación recaería en Becerra. ${ }^{37}$

Las tensiones se agudizaron a raíz del diálogo entablado entre el titular de la UCR, Macario Carrizo y el interventor del PJ, Alberto Serú García. Ello desembocó en la alianza cruzada que protagonizaron las líneas internas de los partidos mayoritarios durante la sesión prevista para votar la necesidad de reformar la constitución. Por un lado, los legisladores de Línea Córdoba y el bloque del peronismo ortodoxo aprobaron en conjunto un despacho; mientras que, por otro lado, los alfonsinistas y el peronismo renovador se expresaron en contra (Closa, 2016:188).

\footnotetext{
36 “Analizan en la UCR la renuncia de Gutiérrez”, $L V I, 1 / 7 / 1986$, p. 5.

37 "Córdoba: se agudiza el pleito del radicalismo", Clarín, 10/8/1986, p. 16.
} 


\section{Constanza Castro}

Si bien ese proceder generó fuertes tensiones internas, Línea Córdoba aseguró el primer paso. De allí en más las negociaciones entre las dos fracciones fueron globales: incluyeron la distribución de los convencionales constituyentes y las candidaturas para la renovación de autoridades del año próximo. Ambos espacios difundieron comunicados en los que expresaban las desavenencias en torno a las posibilidades planteadas en las negociaciones, remitidas a compartir la fórmula gubernamental. Desde el sector alfonsinista consideraban que había existido "poca predisposición" por parte de sus correligionarios, que habían realizado "afirmaciones sujetas a condiciones futuras y de difícil precisión". ${ }^{38}$ Al tratarse el proyecto de ley que convocaba a la elección de Convencionales Constituyentes para el 14 de diciembre, los alfonsinistas se ausentaron en la sesión. Juan José del Boca, senador por esa fracción, justificó la inasistencia como "una demostración de buena voluntad para continuar las tratativas", aspecto que evidenciaba el traslado de los conflictos partidarios a la esfera legislativa de gobierno.

Definido el calendario para las elecciones de convencionales constituyentes, se intensificaron los acercamientos para conformar una lista de unidad. Cada fracción realizó sus nominaciones informándolas luego al titular del partido, quien fue facultado para "compatibilizar" las aspiraciones de todos los sectores internos, sometiéndose en última instancia a consideración de una mesa ampliada del partido. En la distribución, los dos primeros convencionales fueron para Línea Córdoba y el tercero para el MRyC, prosiguiéndose con esa misma estructura de dos y uno en la primera mitad de la nómina, ubicándose algunos representantes del MAY y Línea Nacional en los puestos correspondientes a Línea Córdoba.

Los resultados otorgaron el triunfo al radicalismo que alcanzó poco más del $42 \%$ de los votos; sin obtener mayoría propia, ubicó 37 convencionales sobre un total de 76. ${ }^{39}$ Angeloz había otorgado a la elección un carácter plebiscitario sobre su gestión y dado que no cumplió con las expectativas más optimistas, la reelección no aparecía como un horizonte seguro, alentándose las aspiraciones de Becerra. En sus palabras:

“(...) hice campaña electoral durante cuatro o cinco meses. Yo en ese momento era Secretario General de la Presidencia y un día Alfonsín, que era muy cuidadoso en los problemas internos de las provincias que no eran la suya (...) me llamó y me dijo: 'Mira Carlitos, quiero conversar con vos sobre el problema de Córdoba. Sé de tus aspiraciones, me parece muy bien y además podrás contar con todo mi apoyo personal y político si efectivamente has decidido enfrentarte con Angeloz en una elección interna; pero también te tengo que decir que Angeloz es el gobernador más importante que tiene el país a quien yo le debo respeto, y el Secretario General

\footnotetext{
38 “UCR: instancia crucial en las negociaciones", $L V I, 25 / 9 / 1986$, p. 6

39 “Triunfó la UCR y la renovación del PJ derrotó a la ortodoxia”, $L V I, 15 / 12 / 1986$, p.1.
} 
"La dinámica interna de la Unión Cívica Radical durante la recuperación democrática. Organización partidaria, dirigentes y prácticas políticas (Córdoba, 1983-1987)"

de la Presidencia no puede ser Secretario General y al mismo tiempo enfrentar al gobernador de la provincia de Córdoba' (...)". ${ }^{40}$

La intervención de Alfonsín arbitró en la definición de la candidatura por el ejecutivo provincial, principal nominación en disputa que orientaba las restantes postulaciones. Con la dimisión de Becerra, los máximos referentes de las dos vertientes -reunidos en Alta Gracia- se pronunciaron por la búsqueda de "un camino común". ${ }^{41}$ Éste se materializó en la conformación del binomio para el ejecutivo provincial, cuyo primer término era reservado para Línea Córdoba, descontándose que sería ocupado por Angeloz, quien fue proclamado durante el Foro que su corriente interna realizó el 10 de abril en Villa Giardino, ocasión en la que también se aprobó la incorporación al MRyC. ${ }^{42}$

En el alfonsinismo varios dirigentes se disputaban la nominación a la vicegobernación que finalmente recayó en Mario Negri. Pese a que su nominación fue resistida porque inscribía su militancia en la Capital y se esperaba que la fórmula fuera representativa de la totalidad de la provincia, conformándose con un candidato del interior, su figura cobró prestigio a raíz de su intervención en el levantamiento militar de Semana Santa. En ese entonces, como diputado provincial, estuvo al frente de la Comisión Multisectorial que organizó la marcha en la que coincidieron el gobernador Angeloz junto a su principal opositor, el justicialista José Manuel de la Sota. Además, reunió el apoyo unánime de la totalidad de las seccionales de la capital y exhibía una trayectoria ascendente desde su militancia universitaria en los órganos de cogobierno y partidarios en ámbito juvenil a nivel provincial y nacional, junto a su desempeño en ese entonces- como diputado provincial y vicepresidente primero de la UCR mediterránea que concretaron su postulación. ${ }^{43}$

Junto al binomio del ejecutivo provincial se definieron las nominaciones partidarias. El vicegobernador saliente, Grosso, fue nominado para ocupar la titularidad del partido en representación de Línea Córdoba, secundándolo el dirigente alfonsinista Lucio Prado en la vicepresidencia $1^{\circ}$. Asimismo, la distribución de las secretarías evidenció la representación de la totalidad de las líneas internas, aunque con un marcado predominio de las dos fracciones mayoritarias. A su vez, estas últimas también nominaron, en razón de dos dirigentes cada una, a los delegados que representarían al distrito en el Comité Nacional. ${ }^{44}$

En la Capital, los enfrentamientos entre los dos sectores que se identificaron con Línea Córdoba volvieron a manifestarse. Al igual que Angeloz, Mestre pretendía repetir su mandato en la

\footnotetext{
${ }^{40}$ Entrevista a Becerra, en Frávega, 2006:336.

41 "Coinciden en que no serán necesarias las internas", $L V I, 1 / 3 / 1987$, p. 8.

42 "Postulan a Angeloz para su reelección como gobernador", LVI, 12/4/1987, p. 1.

43 "Nomina Renovación y Cambio el candidato a vicegobernador", $L V I, 11 / 4 / 1987$, p. 1.

${ }^{44}$ Composición del Comité Provincial durante la gestión de Grosso en Frávega, 2006:324.
} 


\section{Constanza Castro}

ciudad, al mismo tiempo que Orgaz instaba por esa nominación contando con el apoyo del gobernador que intentó mediar entre ambos sectores ofreciéndole a Mestre la candidatura por la senaduría nacional. Idénticas gestiones realizó Becerra dado que un sector de su movimiento se involucró en apoyo a la reelección del intendente. Para evitar la contienda, ambos dirigentes propusieron que -en última instancia- la nominación descanse en un tercer candidato surgido del consenso de ambos sectores. Sin embargo, los involucrados firmaron un acta de compromiso en la que se estipulaba la distribución de cargos electivos y partidarios, Mestre retenía la candidatura para la intendencia y Orgaz encabezaba la nómina de diputados nacionales. ${ }^{45}$

Las definiciones adoptadas se relacionaban con las prerrogativas que -nuevamente- el Congreso Provincial había realizado desde mediados del año previo. Estas consistieron en la aprobación formal de la ampliación de su mesa directiva, funcionamiento que en la práctica ya se había adoptado. También dejó en suspenso el artículo que establecía la incompatibilidad en el ejercicio de cargos electivos y partidarios. La irresolución de los principales problemas que aquejaban el funcionamiento del partido fueron fundamentados por la incapacidad de emitir resoluciones ante el desconocimiento de los contenidos de la nueva Constitución. En virtud de ello, la convención partidaria autorizó al Comité central a convocar a los comicios internos en una fecha que se ajustara a la definición de las candidaturas, en tanto la Convención Constituyente se pronunciara sobre la integración del Poder Ejecutivo y Legislativo.

Pautadas para el 7 de junio, las elecciones internas consagraron los acuerdos que las líneas mayoritarias tejieron previamente desde las elecciones para convencionales constituyentes y, particularmente, desde la declinación de Becerra. El ordenamiento vertical que revistió esa acción se reflejó en una notable reducción de la competencia en los niveles inferiores. Sin duda que esa disminución también obedeció -nuevamente- a la imagen de unidad que el partido pretendía brindar frente al asedio de la cuestión militar. No obstante, en unos pocos departamentos fueron realizadas para la selección de senadores provinciales e intendentes. ${ }^{46}$

Cuantitativamente menor, no significaba que los resultados estuvieran exentos de los mismos problemas que caracterizaron la selección de candidatos cuando los acuerdos previos no eran alcanzados. La reglamentación que estipulaba reunir al menos los dos tercios de los votos para acceder a un nuevo mandato despertó una vez más las tensiones entre los radicales. Quienes habían triunfado se negaban a retirar sus candidaturas alegando la legitimidad de la victoria en las urnas; mientras que quienes habían sido derrotados se amparaban en la normativa partidaria y reclamaban para sí la nominación. Frente a esa situación, la resolución final debía ser adoptada por el Congreso Provincial. Los esfuerzos se orientaron a lograr que los candidatos

\footnotetext{
${ }^{45}$ La distribución de los cargos en: Acta de compromiso de candidatos para el departamento Capital de la UCR; "UCR: no habrá interna en la capital", $L V I, 3 / 5 / 1987$, p. 5 .

46 "Interna radical", $L V I, 7 / 6 / 1987$, p. 5.
} 
"La dinámica interna de la Unión Cívica Radical durante la recuperación democrática. Organización partidaria, dirigentes y prácticas políticas (Córdoba, 1983-1987)"

derrotados retiraran sus candidaturas, proclamándose al ganador aún sin obtener los dos tercios. En el caso de los intendentes, de no ser efectiva la primera instancia, se propuso que el candidato surgiera entre los ediles que acompañaron la lista triunfadora. Si bien en algunos casos estas propuestas fueron aceptadas, el conflicto fue más acentuado en torno a la selección de los senadores que generó el pronunciamiento del bloque. En un comunicado dirigido al Comité central solicitaban "una decisión política" favorable para quienes no lograron los dos tercios para la reelección y esgrimía:

“es opinión unánime de los senadores radicales que ese organismo arbitre las medidas necesarias a efecto de que se respete en todos los casos la expresión mayoritaria volcada en las urnas el 7 de junio y, en consecuencia, se proceda a la proclamación de todos aquellos candidatos a cargos electivos que hayan obtenido la mayoría de los votos, no importando el porcentaje alcanzado (...) "[resulta necesario] mantener en la función pública y en los máximos niveles partidarios a los dirigentes con mayor representatividad y que hayan dado muestras de competencia en el desempeño de la función (...) el mantenimiento de la normativa del Art. 135 de la carta orgánica no hace sino encubrir una proscripción". ${ }^{47}$

En relación a estos reclamos, el Comité Provincial decidió la conformación de una comisión cuyo misión consistió en arribar a una "solución política". ${ }^{48}$ En definitiva, facultaba ad hoc a esa comisión a legitimar una práctica usual en las resoluciones de las nominaciones cuando estas no podían resolverse tal como lo reglamentaba la carta orgánica.

Finalmente, las elecciones del 6 de septiembre de 1987 otorgaron nuevamente el triunfo a la UCR, esta vez con un margen notablemente reducido que contrastaba con los desempeños previos. Al superar a su rival por tan solo cinco puntos porcentuales, obtuvieron mayoría en ambas cámaras legislativas, con una marcada diferencia en senadores y repitieron el éxito en numerosas intendencias, aunque significativamente menor. ${ }^{49}$

Sin embargo, el ajustado triunfo cobró relevancia nacional a la luz del fuerte retroceso que sufrió el partido. Además de Córdoba, la UCR solo retuvo la gobernación de Río Negro. Los balances prestigiaron al radicalismo cordobés y los objetivos inmediatos apuntaron a fortalecer su representación en el Comité Nacional que debía renovar sus autoridades a principios de diciembre. Ahora, la estrategia no consistió en alcanzar una mayor representación mediante la

\footnotetext{
47 "Proclaman los candidatos y autoridades de la UCR", LVI, 26/6/1987, p. 4.

48 "UCR: gestiones por la reelección de candidatos", $L V I, 30 / 6 / 1987$, p. 6.

${ }^{49} \mathrm{La}$ UCR contó con 40 senadores y 36 diputados provinciales y 5 diputados nacionales. A nivel local, triunfó en 143 comunas, perdiendo el gobierno de 27 comunas. "Difundieron los cómputos oficiales de la elección”, LVI, 2/10/1987, p.4.
} 


\section{Constanza Castro}

incorporación a una vertiente de proyección nacional sino destacando la singularidad del radicalismo mediterráneo.

El tiempo que medió hasta la asunción de las nuevas autoridades nacionales evidenció una reconfiguración interna. La figura de Angeloz cobró prestigio a nivel nacional posicionándose en la carrera presidencial de 1989, aun cuando no había asumido su segundo mandato como gobernador. Tan es así que un grupo de radicales impulsó tempranamente su candidatura en noviembre de ese mismo año, pronunciándose en el mismo sentido otros nucleamientos y dirigentes. ${ }^{50}$ A la vez, en la renovación de autoridades nacionales del partido, Alfredo Orgaz ocupó la vicepresidencia segunda. De esa manera, se cumplió con uno de los objetivos que -a corto plazo- se había propuesto la dirigencia cordobesa en los últimos tiempos. Sin embargo, la individualidad continuó siendo el camino elegido para alcanzar mayor gravitación. El alfonsinismo, si bien había crecido en los años de gobierno, no pudo afianzarse internamente ni ampliar sus bases de sustentación. Por el contrario, para quienes militaron en las filas de Línea Córdoba, los resultados rectificaron el rumbo que históricamente habían adoptado al dar cuenta de la individualidad que la provincia representó en el concierto partidario nacional. De allí que, más que insertarse en una vertiente interna de alcance nacional, el objetivo consistió en imponer desde Córdoba una nueva corriente que asegurase su predominio, materializándose en la emergente Línea Federal que llevó - posteriormente- como “candidato natural” a Angeloz a disputar la presidencia.

\section{Recapitulación final}

Sin renunciar a la dimensión nacional de la política argentina, hemos adoptado una perspectiva de estudio a escala subnacional que permitió complejizar el análisis sobre el desempeño partidario de la UCR en la provincia de Córdoba. La selección respondió a las transformaciones que caracterizaron a la política argentina desde la recuperación democrática, acentuándose el protagonismo de las provincias como ámbitos de concentración de apoyos para lanzarse a la competencia nacional.

El recorrido evidenció la especificidad del radicalismo provincial en cuanto a las líneas internas que nutrieron el partido. En la apertura electoral de 1983, Línea Córdoba presentó una estructura consolidada y reunió dirigentes con trayectorias y bases territoriales para asegurarse el control de los espacios de conducción partidaria y gubernamental. En ese escenario, los militantes del MRyC se incorporaron como socios minoritarios de la alianza. Al examinar el funcionamiento

\footnotetext{
50 Edison Otero, Osvaldo Álvarez Guerrero y Juan Carlos Pugliese se refirieron a Angeloz como el "candidato natural" del radicalismo para reemplazar a Alfonsín. "Expresiones de respaldo a la candidatura de Angeloz", Clarín, 25/10/1987, p.7; "Postulan a Angeloz", LVI, 19/11/1987, p. 6.
} 
"La dinámica interna de la Unión Cívica Radical durante la recuperación democrática. Organización partidaria, dirigentes y prácticas políticas (Córdoba, 1983-1987)"

del partido, identificamos un conjunto de lógicas de interacción que caracterizaron a la organización y los problemas que orientaron sus acciones. Específicamente destacamos tres nudos principales: la relación partido-gobierno, la inserción partidaria en la estructura nacional y los conflictos intrapartidarios en torno a las nominaciones. En relación al primero, el cuestionamiento sobre la inmovilización del partido o la confusión entre partido y gobierno actuó como un recurso utilizado por los nucleamientos en coyunturas específicas marcadas por la relativa marginalidad en la representación partidista, o bien cuando se lanzaban a la competencia para invalidar a su adversario.

El segundo eje encerraba una preocupación más amplia. En su empeño por lograr una mayor gravitación en la conducción nacional, Línea Córdoba promovió su inserción en el MRyC. Ese objetivo puso en debate dos aspectos: por un lado, confrontó dos visiones sobre la historia reciente del radicalismo provincial, particularmente de la alianza en las elecciones internas de 1972 interpretada por el MRyC como circunstancial, y evocada por Línea Córdoba como una reinserción; por otro lado, el liderazgo constituyó un obstáculo en tanto las figuras de Becerra y Angeloz se presentaban como imposibles de compatibilizar en una nueva corriente interna.

Subyacente al tercer eje se develó la existencia de una serie de prácticas informales que se expresaron en las definiciones de las nominaciones. En general, los acuerdos comenzaban con la negociación entre los dirigentes de las principales fracciones, continuaban con la elección de candidatos por parte de cada una de ellas y, una vez completado ese recorrido, eran convalidadas en las instancias orgánicas como una mera formalidad. En ese proceso, los conflictos se suscitaron en los cargos de menor relevancia y las elecciones internas fueron efectivas en instancias locales. La dosis de informalidad también se evidenció en las resoluciones que los órganos partidarios debían implementar en relación a las incompatibilidades en el desempeño de los cargos públicos y partidarios como también en las definiciones sobre las reelecciones. En el primer caso apeló a numerosas prórrogas; mientras que, en el segundo los dirigentes intentaban resolver las situaciones conflictivas involucrándose en ellas y, al no lograr un resultado favorable, el partido creaba comisiones que legitimaban ese accionar previo. Siempre las prácticas políticas informales antecedieron la acción normativa y/o resolutiva del partido.

En la construcción de la hegemonía partidaria, para Línea Córdoba retener la gobernación constituía su objetivo primordial. En consecuencia, negoció con los sectores ortodoxos del peronismo para lograr la aprobación de la necesidad de la reforma constitucional. En concomitancia con la informalidad de las negociaciones de las candidaturas, la intervención de Alfonsín terminó arbitrando la nominación mayor, y ello ordenó las restantes. Esa resolución 


\section{Constanza Castro}

evidenció la supremacía del rol de gobernador al neutralizar a su principal rival afincado en otro espacio institucional en el orden nacional.

Los resultados de las elecciones de septiembre de 1987 significaron un quiebre en las perspectivas trazadas. La derrota a nivel nacional del radicalismo puso freno al impulso alfonsinista que atravesó una gran conmoción interna. A su vez, la derrota coartó la posibilidad de que Línea Córdoba se incorpore al MRyC. Más bien, la vertiente buscó diferenciarse y acentuar aún más su perfil individual. La figura de Angeloz se proyectó como sucesor de Alfonsín dado que, de los dos distritos en los que la UCR había retenido el triunfo, Córdoba era el más gravitante en términos electorales.

\section{Bibliografía}

Cavarozzi, M. (2006). Autoritarismo y democracia (1955-2006). Buenos Aires: Ariel.

Closa, G. (2016). Córdoba. En: M. Ferrari y V. Mellado, (comps.), La renovación peronista. Organización partidaria, liderazgos y dirigentes 1983-1991. Sáenz Peña: EDUNTREF.

De Luca, M.; Jones, M. y Tula, I. (2008). Revisando las consecuencias políticas de las primarias. Un estudio sobre las elecciones de gobernador en la Argentina. Revista Postdata, v. 13, n. 2, 81-102.

Delgado, M. S. (2003). "El otro partido. Algunas consideraciones acerca del radicalismo (19831989)", en VI Congreso Nacional de Ciencia Política. Rosario, UNR.

Frávega, O. (2006). Historia de la Unión Cívica Radical de Córdoba 1890/2000. Córdoba: Editorial Brujas.

Persello, V. (2007). Historia del radicalismo. Buenos Aires: Edhasa.

Sartori, G. (2005 [1976]). Partidos y sistemas de partidos. Marco para un análisis. Madrid: Alianza.

Tcach C. (1991). Sabattinismo y peronismo: partidos politicos en Córdoba, 1945-1955. Buenos Aires: Sudamericana.

Tula, M. I. y De Luca, M. (2012). Reglas electorales y dinámicas políticas en la selección de candidatos. Cambios y continuidades de Alfonsín a los Kirchner. En A. Malamud y M. De Luca (coords.), La política en tiempos de los Kirchner. Buenos Aires: Eudeba.

Recepción: 22/06/2021

Evaluado: 17/10/2021

Versión Final: 19/11/2021 